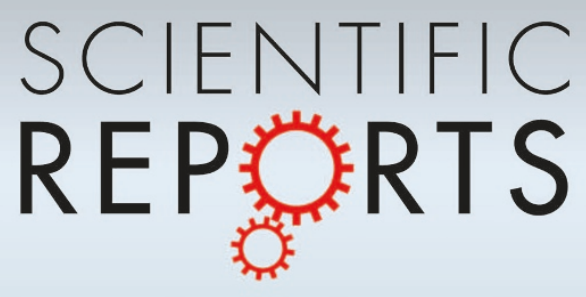

OPEN

SUBJECT AREAS: STRUCTURAL PROPERTIES MECHANICAL PROPERTIES CHARACTERIZATION AND ANALYTICAL TECHNIQUES

METALS AND ALLOYS

Received 16 April 2013

Accepted

10 June 2013

Published

3 July 2013

Correspondence and requests for materials should be addressed to

J.L. (liju@mit.edu) or

Z.-W.S. (zwshan@ mail.xjtu.edu.cn)

\section{Visualizing size-dependent deformation mechanism transition in $\mathrm{Sn}$}

\author{
Lin Tian', Ju Li ${ }^{1,2}$, Jun Sun', Evan Ma ${ }^{1,3} \&$ Zhi-Wei Shan'
}

${ }^{1}$ Center for Advancing Materials Performance from the Nanoscale (CAMP-Nano) \& Hysitron Applied Research Center in China (HARCC), State Key Laboratory for Mechanical Behavior of Materials, Xi'an Jiaotong University, Xi'an 710049, P. R. China, ${ }^{2}$ Department of Nuclear Science and Engineering and Department of Materials Science and Engineering, MIT, Cambridge, Massachusetts 02139, USA, ${ }^{3}$ Department of Materials Science and Engineering, Johns Hopkins University, Baltimore, Maryland 21218, USA.

Displacive deformation via dislocation slip and deformation twinning usually plays a dominant role in the plasticity of crystalline solids at room temperature. Here we report in situ quantitative transmission electron microscope deformation tests of single crystal Sn samples. We found that when the sample size was reduced from $450 \mathrm{~nm}$ down to $130 \mathrm{~nm}$, diffusional deformation replaces displacive plasticity as the dominant deformation mechanism at room temperature. At the same time, the strength-size relationship changed from "smaller is stronger" to "smaller is much weaker". The effective surface diffusivity calculated based on our experimental data matches well with that reported in literature for boundary diffusion. The observed change in the deformation mode arises from the sample size-dependent competition between the Hall-Petch-like strengthening of displacive processes and Coble diffusion softening processes. Our findings have important implications for the stability and reliability of nanoscale devices such as metallic nanogaps.

ased on how atomic registries change during the plastic flow, deformation mechanisms of solids can be classified into three main classes: displacive, diffusional, and hybrid (coupled displacive and diffusional). Displacive deformations, e.g. dislocation glide and deformation twinning, tend to be prevalent at low temperature; the atomic registries change in a collective and semi-deterministic manner. In contrast, diffusional plasticity, e.g. Nabarro-Herring or Coble creep ${ }^{1}$, often occurs at high temperature; concurrent with the deformation the atomic registries are randomized in an atom-by-atom manner ${ }^{2}$. Aside from the temperature, size scale can also be a decisive factor on the deformation mechanism ${ }^{3-9}$. Recently, Strachan et al.$^{10}$ found that two singlecrystalline Au nano-electrodes (tip radius of curvature $R \sim 10 \mathrm{~nm}$ ) receded by about $10 \mathrm{~nm}$ after sitting at room temperature for 4 months. This suggests that nano-structured devices may lose their long-term shape stability due to diffusion as the characteristic size goes down to nanometric scale. Therefore, for the reliability and stability of nano-structured devices, e.g. molecular electronics ${ }^{11}$, nanoscale break junction ${ }^{12,13}$, it is critical to investigate the size-diffusion-strength relationship. However, little has been done in the literature on this topic in a quantitative manner.

In this work, we take advantage of the quantitative nanomechanical test and in situ Transmission Electron Microscopy (TEM) to demonstrate surface-to-grain boundary (GB) diffusional creep (Coble creep ${ }^{1}$ ) in nanoscale materials. We purposefully chose $\mathrm{Sn}$, a low melting point $\left(T_{\mathrm{M}}=505 \mathrm{~K}\right)$ metal, for which the homologous temperature $\left(T_{\mathrm{H}} \equiv T / T_{\mathrm{M}}\right)$ is high $\left(T_{\mathrm{H}} \approx 0.6\right)$ even at room temperature. This facilitates the observation of the size-induced switch to diffusional mechanism at room temperature, without the need for heating using specialized holders in TEM or months of waiting in the mechanical tests.

\section{Results}

We begin with relatively large samples, with their diameters ranging from 450 to $500 \mathrm{~nm}$. The compression tests showed that their plastic deformation process is characterized by sudden strain burst and drastic load drop (Fig. 1). For simplicity, we will only address the sample marked with black color in details. Kikuchi diffraction pattern demonstrated the tested sample which has $D=450 \mathrm{~nm}$ is initially single crystal. Fig. $1 \mathrm{~b}$ and Fig. 1c are still frames extracted from the recorded movie and correspond to the points marked with $b$ and $c$ in Fig. 1a, respectively. The stress increased linearly with strain up to about $300 \mathrm{MPa}$ (point b in Fig. 1a). Following this, a sudden strain burst occurred. At the same time, the force sensed by the loading cell dropped to zero (point $c$ in Fig. 1a). During the strain burst, obvious geometry change occurred (Fig. 1c). Slip step, characteristic of displacive deformation, can be identified clearly by postmortem scanning electron microscope (SEM) examination (Fig. 1d). 
a

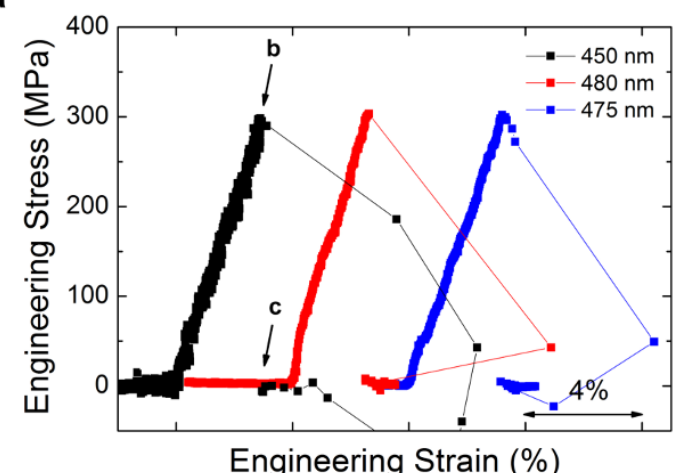

b

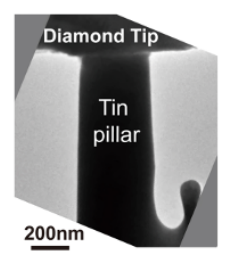

C

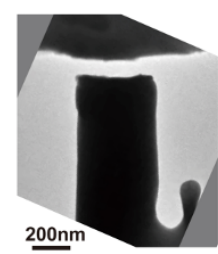

d

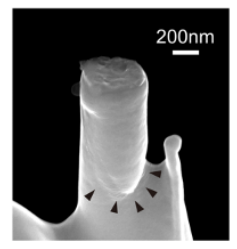

Figure $1 \mid$ Compression test of Sn pillars with diameter larger than $450 \mathrm{~nm}$. (a) Engineering stress-strain curves from the compression tests, with average strain rate of $\sim 5 \times 10^{-3} / \mathrm{s}$. (b) and (c) are TEM bright field images of the pillar before and after its collapse, representing the moments marked in the stress-strain curve for the sample with diameter of $450 \mathrm{~nm}$ (black curve). (d) Post-mortem scanning electron microscopy (SEM) image of the $450 \mathrm{~nm}$ pillar showing the slip step.

The flat punch velocity was set to be $20 \mathrm{~nm} / \mathrm{s}$ and the corresponding strain rate is about $5 \times 10^{-3} / \mathrm{s}$. The data acquisition rate used for this test was 200 points per second which means that the interval between every two data points was 0.05 second. A conservative evaluation suggests that the maximum strain rate during the strain burst is as high as $\sim 0.9 / \mathrm{s}$, another indication of displacive deformation. In addition, the apparent yield stress ( $\sim 300 \mathrm{MPa}$, point b in Fig. 1a) prior to plastic deformation (the strain burst) is remarkably high for Sn, since the yield strength of bulk $\mathrm{Sn}$ is only about $9 \sim 15 \mathrm{MPa}^{14}$. The elevated strength for submicron samples is consistent with the well-established tenet, i.e. smaller is stronger. Similar elastic-plastic behavior has been reported for other submicron-sized metals ${ }^{15-18}$. Apparently, displacive mechanism still dominates the plastic deformation at room temperature for Sn samples in this size regime.

Remarkable changes in deformation behavior occurred when the sample size was reduced below $230 \mathrm{~nm}$ or so, as shown in Fig. 2a. Significant plastic flow set in at lower yield stress (defined by loss of stress-strain proportionality) or even without a well-defined yield point. For example, the stress-strain curve of the sample with $D=$ $230 \mathrm{~nm}$ (black curve in Fig. 2a) is quite continuous except that a mild load drop occurred when the engineering strain reached $\sim 10 \%$ (point $d$ in Fig. 2a). Fig. 2b-d are still frames extracted from the recorded movie that correspond to the points marked in Fig. 2a, respectively. A small slip step was created on the tested sample (marked with black arrow heads in Fig. 2d) during the mild load drop. For the sample with $D=200$ nm (blue curve in Fig. 2a), even though the load drop is similar to that observed in Fig. 1a, the yield stress and amplitude of the strain burst are obviously lower. The lowered, blurred yield point and the large continuous plastic flow observed in these samples are in sharp contrast with those observed in larger pillars (Fig. 1), namely obvious yield point, sudden and dramatic load drop and strain burst. This difference suggests a change in the deformation mode due to the decreased sample size.

It becomes difficult to directly FIB-fabricate samples with $D<$ $150 \mathrm{~nm}$, because of the relatively low melting point of Sn. However,

a



b

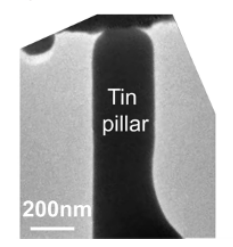

C
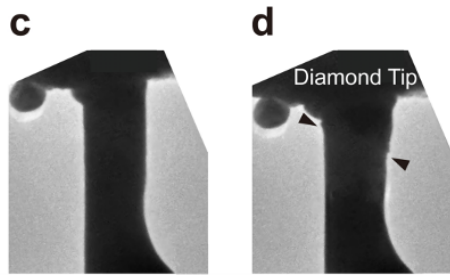

Figure $2 \mid$ Compression test of Sn pillars with diameter smaller than $230 \mathrm{~nm}$. (a) Engineering stress-strain curves from the compression tests, with average strain rate of $\sim 5 \times 10^{-3} / \mathrm{s}$. (b)-(d) Still frames extracted from the recorded movie correspond to the events marked in the stress-strain curve for the sample with diameter of $230 \mathrm{~nm}$ (black curve).

we found that after first compressing a larger pillar sample to "pancake" shape, the heavily deformed Sn adhered to the flat diamond punch. Subsequent pulling (switching from compressive to tensile loading) forms a narrower- $D$ ligament, providing a means to observe the mechanical behavior of smaller- $D$ samples. Compared with FIBed samples, these samples had fresh surfaces (no oxides generated in the high vacuum inside the TEM) like the mechanical break junctions ${ }^{12,13}$ and were free of FIB damage. One of the "tensile" examples is shown in Fig. 3: after heavy compression, good mechanical contact forms between the sample and diamond tip. By pulling back the diamond tip, a ligament with a neck was created. Movie and images were taken under dark field condition using diffraction spot indicated by the white arrow in Fig. 3b. Grain boundaries (GBs) were observed to form through dynamic recrystallization during the aforementioned pre-deformation process: a region with bright contrast, meaning Bragg's law is satisfied, appeared in the neck, where the single-crystal ligament height (the grain size) is seen to be on the same order as its diameter $D$ (Fig. 3d). As the tip withdraw, the necked part got thinner and thinner (Fig. 3d-e), and broke apart at $t$ $=2.6 \mathrm{~s}$ (Fig. 3f). Interestingly, instead of forming chisel-like morphology as frequently observed in displacive deformation ${ }^{19-21}$, the fractured tips shrunk and receded quickly after broken.

Subsequent quantitative tensile test of a nanoscale ligament also suggested that diffusional plasticity plays a dominant role at room temperature, as shown in Fig. 4. The probe velocity and the video recording rate for this test were $10 \mathrm{~nm}$ per second and 5 frames per second, respectively. Fig. $4 \mathrm{a}-\mathrm{d}$ are still frames extracted from the recorded movie (see Supplementary Movie 1) showing the evolution of the sample. The starting minimal diameter of this sample $\left(D_{\min }\right)$ is about $130 \mathrm{~nm}$ (Fig. 4a), which decreased continuously (Fig. 4a-c) in response to the externally applied tensile force. At $t=5.0$ second, the sample was seen to be torn into two parts (Fig. 4d). Again, instead of leaving behind chisel like sharp tips, the two parts exhibited quite rounded and smooth geometry. This means that in less than 0.2 second (see Fig. $4 \mathrm{c}$ and $4 \mathrm{~d}$ ), the fractured sample receded into the geometry as shown in Fig. $4 \mathrm{~d}$ through diffusional deformation. The morphology evolution is very similar to that of liquid drop. However, 

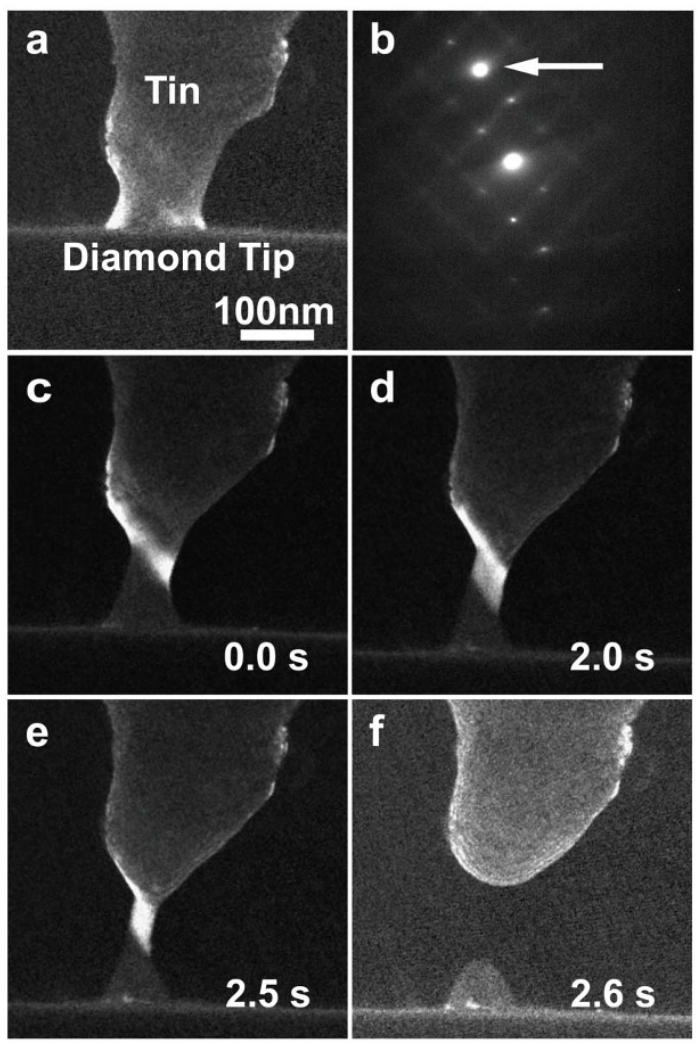

Figure 3 Dynamic recrystallization and grain boundary plating out during the tensile deformation of a nanoligament. (a) The sample profile prior to the tensile test. (b) Selected area diffraction pattern showing the sample orientation and imaging condition. (c-d) Dynamic evolution evidenced the formation of the new grain and deformation process of the nanoligament. (e) Final morphology of the fractured nanoligament.

both the mechanical data (Fig. 4e) and high resolution TEM observation of the sample surface (See Supplementary Movie 2) suggested that the samples were still crystalline solids.

One advantage of in situ test is that the projected diameter of the samples can be recorded and then measured with high accuracy (Fig. 4e, half-filled blue circles). By assuming a circular cross section with minimal diameter $D_{\min }$, the corresponding true stress vs. time curve is plotted in Fig. 4e (black dots). The time points that correspond to the frames shown in Fig. $4 \mathrm{a}-\mathrm{d}$ are marked with black arrows in Fig. 4e. It is worth noting that the apparent yield strength ( $\sim 60 \mathrm{MPa})$ of this $130 \mathrm{~nm}$ sample, as defined by loss of stress-strain proportionality, is 5 times lower than that observed in the $450 \mathrm{~nm}$ sample (Fig. 1a). This "smaller is weaker" observation is very different from the "smaller is stronger" trend in all previous reports for metals (with higher melting temperatures) with similar sizes ${ }^{16,22-26}$, and is presumably due to the significant contribution from the diffusional plasticity: atom transport along the free surface, and plating out at the $\mathrm{GBs}^{27-29}$.

The strain hardening trend in Fig. $4 \mathrm{e}$ after $t=2.5$ second can be rationalized by the increasing separation from atom source (surface) to atom sink (GB) and by the increase of the local strain rate. Along with the tensile deformation, necking of the sample will inevitably create cone-like geometry. This will generate stress gradient and therefore lead to localized plastic deformation. As mentioned earlier in this paper, the tensile test was carried out under displacement rate control, i.e. under fixed probe velocity. Consequently, the local strain rate of the necking part will keep increasing rather than being constant along with plastic deformation. This was supported by the increasing shrinking rate of the minimum diameter $D_{\text {min }}$ of the tested sample (Fig. $4 \mathrm{a}-\mathrm{c}$ and e). By taking the derivative of $D_{\min }$ with respect a

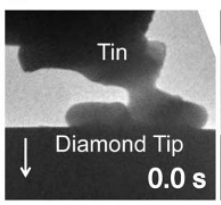

b

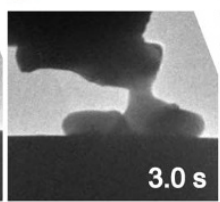

C

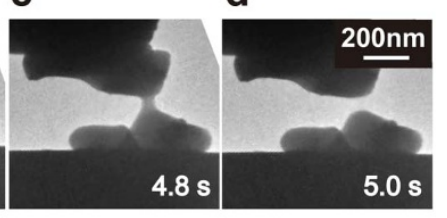

e

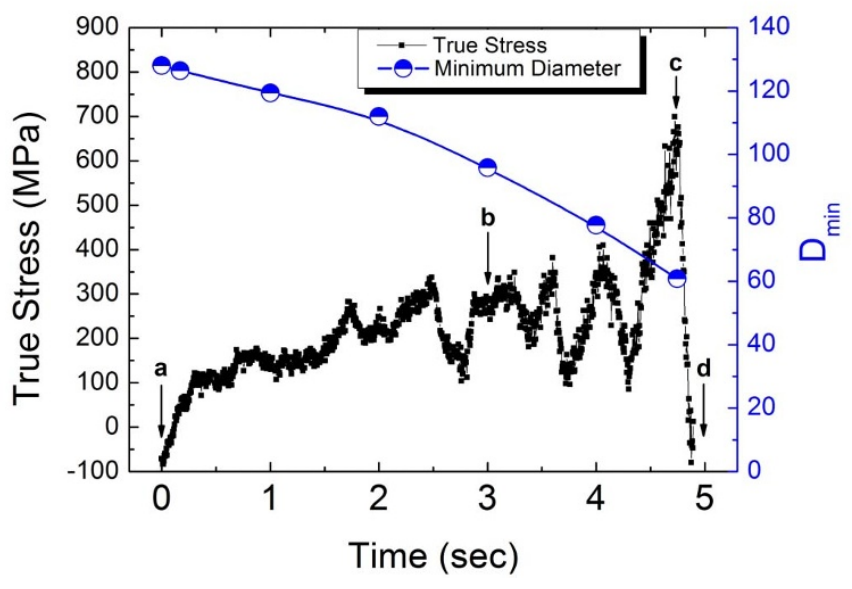

Figure $4 \mid$ Tensile test of a $130 \mathrm{~nm}$ Sn nanoligament. (a-d) Still frames showing the deformation process of the Sn nanoligment. (e) The maximum stress plotted as a function of time (black). Instantaneous minimum diameters of the sample measured from the still frames were also plotted (blue color). Note the negative stress is due to the switch from compression to tension.

to time $t$, we found that $d D_{\min } / d t$ was about $5 \mathrm{~nm} / \mathrm{s}$ at the beginning of this tensile test. However, this value increased almost 5 times just prior to the fracture of this tensile sample. Therefore, the strain hardening trend agrees well with the strain-rate sensitive nature of diffusional deformation mechanism. Several major stress serrations occurred after 2.5 seconds. This suggested that some displacive plasticity might also accompany the diffusion-dominant deformation; the increasing serration amplitude in true stress may indicate the "quantum" nature of displacive plasticity, namely, a single dislocation slip-out can cause larger and larger strain and stress relaxation when averaged over smaller neck volume $\propto D_{\min }{ }^{3}$. However, no surface slip steps were observed during the deformation of the nano-ligaments. This is in sharp contrast with those observed in typical displacive deformation. For example, strained sub-10-nmsized gold crystals always have terraces or staircases on the surface of the material ${ }^{19-21}$. Assuming that displacive deformation (e.g. dislocation burst) did take place in our test and generate terraces on its surface, because of the overwhelming diffusional deformation for the given sample size, the terraces resulted from displacive deformation would be smoothed out quickly by surface diffusion.

\section{Discussion}

Ohring et al. reported the temperature-dependent diffusivity of Sn. At $300 \mathrm{~K}$, bulk diffusivity ${ }^{30}$ and grain boundary ${ }^{31}$ diffusivity of $\mathrm{Sn}$ are $2.86 \times 10^{-17} \mathrm{~m}^{2} / \mathrm{s}$ and $1.45 \times 10^{-12} \mathrm{~m}^{2} / \mathrm{s}$, respectively. Compared with grain boundary, free surface has reduced constraints and therefore should have even larger diffusivity. Consequently, the surface layer may serve as a highway for short-circuit mass transport. The contribution of the surface diffusional plasticity to the overall plasticity should increase with decreasing sample size. Based on these considerations, we have constructed models to explain the observed diffusional deformation in this work, as shown in Fig. 5. We assumed 
that a disordered surface layer ${ }^{32}$ exists on the single-crystalline sample surface and its nominal thickness $\delta_{\mathrm{s}}$ is $\sim 1 \mathrm{~nm}$. The surface chemical potential $(\mu)$ controls the drift of the surface atoms. This surface chemical potential is a function of external applied stress, surface tension, and sample geometry. If no stress is applied to the ligament, as shown in Fig. 5a mimicking samples in both Fig. 3 and Fig. 4, surface atoms will flow from the thicker parts (high $\mu$ ) to the neck $($ low $\mu$ ). As a result the valley is filled and the neck grows thicker and the whole ligament becomes more uniform, see Fig. 5a-5b. Fig. $5 c-5 d$ describe a possible scenario occurred in the real samples shown in Fig. 3 and Fig. 4. The applied stress increased the elastic energy in the sample, in particular the necking part, such that the chemical potential $(\mu)$ in the neck is even higher than the other parts. Diffusion flow is reversed and thus the neck is getting thinner and thinner. The presence of grain boundaries as a result of heavy deformation and recrystallization contributes to the plastic deformation by accommodating the large plastic flow through GB diffusion and plating out. Specifically, the chemical potential gradient drives diffusional flow of atoms to plate into the GBs (see Fig. $5 c-5 d$ ), mediating the specimen elongation under the tensile stresses.

Tensile stress is released when the neck breaks. The fractured surfaces of the just broken sample have the geometry of the small hills shown in Fig. 5e. The hills however smooth out within just one video frames, 0.2 second at most. This process is driven by surface tension only, i.e., with no external stress at play. As shown in Fig. 5e, the chemical potential of point $x$ in the surface layer is $\mu(\mathbf{x})=\frac{\gamma \Omega}{R(\mathbf{x})}, \gamma$ is the surface energy, $\Omega$ is the atomic volume of $\mathrm{Sn}$, and $R(\mathrm{x})$ is radius of curvature. Assuming atoms in the surface layer perform random walk, the resulting mass flux $J$ across the surface layer is,

$$
J_{\mathrm{s}}=-\frac{X_{\mathrm{s}}}{\Omega} \delta_{\mathrm{s}} \frac{D_{\mathrm{s}}}{k_{\mathrm{B}} T} \partial_{s} \mu,
$$

given that the mobility of atom is related to diffusivity by Einstein relation $^{33}$. In Equation (1) $X_{s}$ is the solubility of diffusing atom in surface liquid layer, $\delta_{s}$ is the nominal surface layer thickness, $k_{\mathrm{B}}$ is Boltzmann constant, $T$ is temperature, and $D_{s}$ is surface diffusivity of $\mathrm{Sn}$. According to the law of conservation of mass, the deposition rate:

$$
\partial_{t} h+\Omega \partial_{s} J_{s}=0 .
$$

This leads to

$\partial_{t} h=-\Omega \partial_{s} J_{\mathrm{s}}=\frac{X_{\mathrm{s}} \delta_{\mathrm{s}} D_{\mathrm{s}}}{k_{\mathrm{B}} T} \partial_{s}^{2} \mu=\frac{\gamma \Omega X_{\mathrm{s}} \delta_{\mathrm{s}} D_{\mathrm{s}}}{k_{\mathrm{B}} T} \partial_{s}^{2} \frac{1}{R(s)} \sim \frac{\gamma \Omega X_{\mathrm{s}} \delta_{\mathrm{s}} D_{\mathrm{s}}}{k_{\mathrm{B}} T} \frac{1}{R_{0}^{3}}$
As

$$
\partial_{t} h \sim \frac{h_{0}}{\tau},
$$

Where $\tau$ is the timescale of the neck evolution, we have

$$
\frac{h_{0}}{\tau} \sim \frac{\gamma \Omega X_{\mathrm{s}} \delta_{\mathrm{s}} D_{\mathrm{s}}}{k_{\mathrm{B}} T} \frac{1}{R_{0}{ }^{3}} .
$$

Therefore,

$$
D_{\mathrm{s}} \sim \frac{h_{0} R_{0}{ }^{3} k_{\mathrm{B}} T}{\tau \gamma \Omega X_{\mathrm{s}} \delta_{\mathrm{s}}}
$$

Suppose $\tau=0.2 s, h_{0}=1 \times 10^{-7} \mathrm{~m}, R_{0}=5 \times 10^{-8} \mathrm{~m}, X_{s}=1$, we get $D_{\mathrm{s}}$ $\sim 8 \times 10^{-12} \mathrm{~m}^{2} / \mathrm{s}$. This value is on the same order of that reported previously for grain boundary diffusivity in $\mathrm{Sn}^{31,34,35}$, which means that the actual diffusion ability of $\mathrm{Sn}$ at $300 \mathrm{~K}$ is able to achieve the observed deformation rate. Since the boundary diffusivity limits the amount of mass transport, diffusion deformation is only prominent when sample size is small enough and the strain rate is not very high, depending on the material and the deformation conditions.

The plastic deformation mechanisms of crystalline solids have been mapped by Ashby using the so-called deformation-mechanism $\operatorname{map}^{36}$. Depending on the applied strain rate $(\dot{\varepsilon})$ and stress $(\sigma)$, the homologous temperature $\left(T_{\mathrm{H}}\right)$ and the characteristic size scale $(D)$ of the component ${ }^{2,37}$, a specific mechanism will play a dominant role to carry out the plastic deformation. Normally, displacive mechanism will dominate at lower homologous temperatures but requires higher stress. Diffusive mechanism, in contrast, will only be prevalent at high homologous temperatures, but needs lower stress. The key message from our work here is that the regime boundaries of the deformation-mechanism map indeed shift with reducing sample $\operatorname{size}^{2,37}$.

For materials deformed through displacive mechanisms, such as dislocation slip or deformation twinning, it has been well established that the yield stress follows a Hall-Petch size-strengthening relationship, i.e. $\sigma=\sigma_{0}+k D^{-\alpha}$, where $\alpha$ is typically between $1 / 2$ and $1^{23,38}$. The diffusional deformation, e.g. Coble creep, is strongly timedependent (very high strain-rate sensitivity), without a sharply defined threshold stress as in displacive yielding. The strain rate due to Coble creep ${ }^{1}$ is expected to be

$$
\dot{\varepsilon}_{\text {Coble creep }}=K \frac{\delta_{\mathrm{s}} D_{\mathrm{s}} \Omega}{D^{3} k_{\mathrm{B}} T} \sigma,
$$

where $K$ is a dimensionless constant. Equation (7) is of the same origin as Equation (6). For a fixed strain rate, one can see that $\sigma$

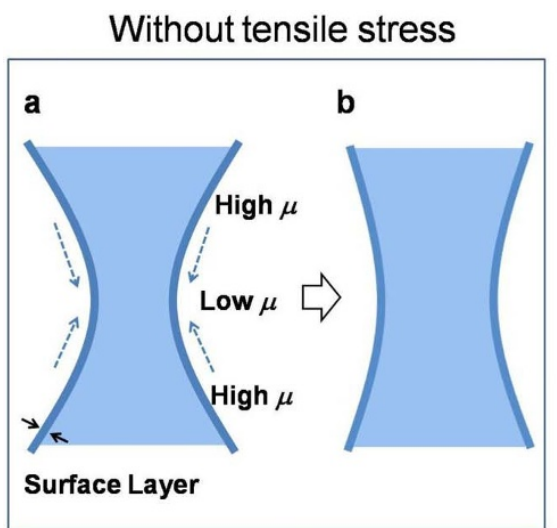

With tensile stress, and two GBs

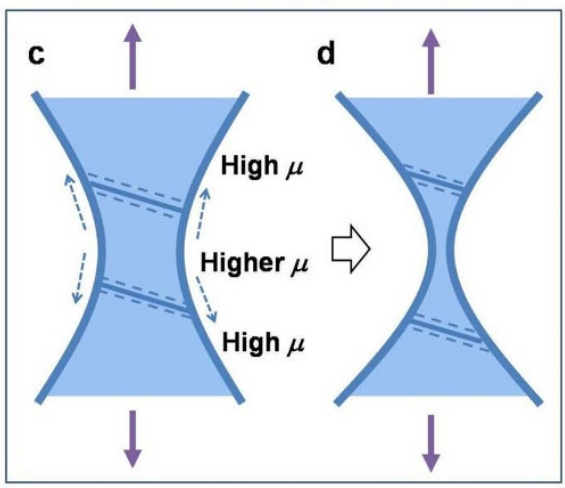

After fracture

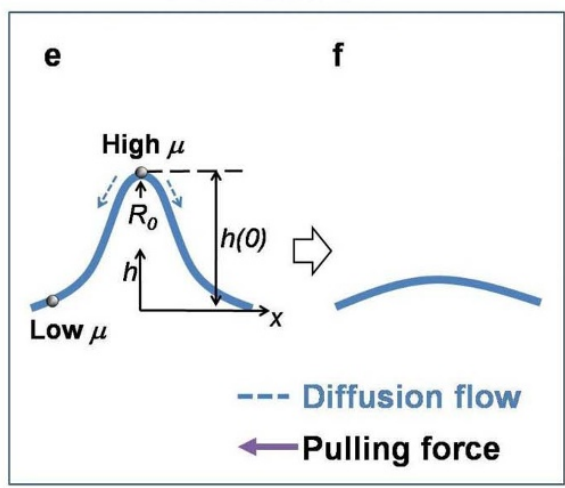

Figure $5 \mid$ Schematic of a diffusion dominant deformation model for the nanoligament. (a)-(b) Without tensile stress curvature driven diffusion flow will smooth out the necked area in the ligament leaving a ligament with more uniformed cross section (b). (c)-(d) Tensile stress increased the chemical potential in the thinnest part to overcome the chemical potential gradient caused by curvature. The two boundaries are sinks to the atom flow. (e)-(f) Sample profile evolution after fracture. 


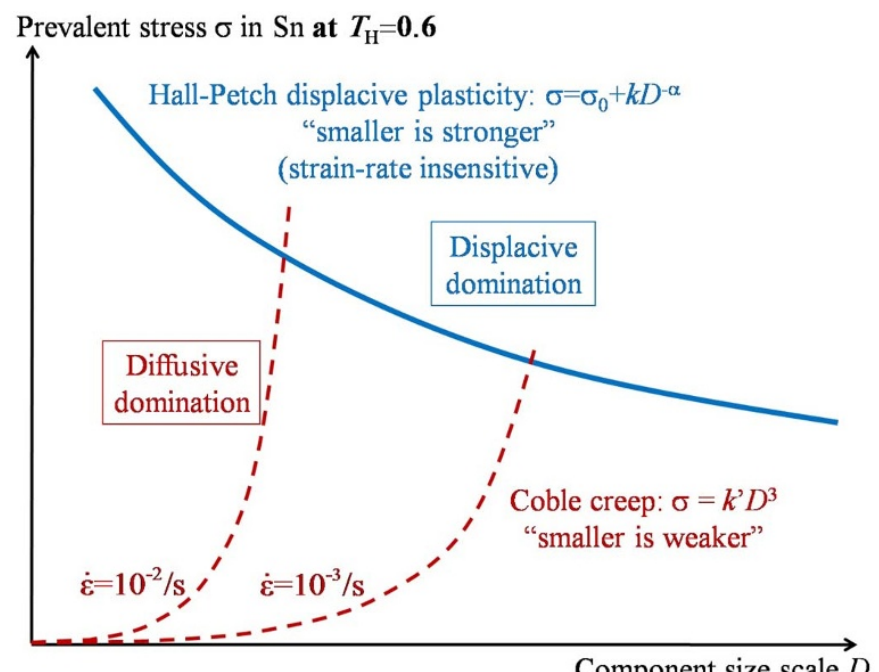

Component size scale $D$

Figure 6 | Semi-quantitative illustration of deformation mechanism map of $\mathrm{Sn}$. For displacive deformation, Hall-Petch strengthening predicts "smaller is stronger" trend (red curve). Surface diffusional deformation is governed by Coble creep. At a fixed strain rate, stress follows $\sigma \propto D^{3}$, which shows "smaller is weaker" (black curve). These two curves crossed and distinguished the two deformation mechanism, i.e. displacive plasticity, and diffusive plasticity.

$\propto D^{3}$. Thus, "smaller is much, much weaker" for Coble creep. Along with the reducing sample size, the competition between "smaller is stronger" displacive yield and "smaller is much weaker" Coble creep lead to an interesting crossover, as illustrated in Fig. 6. The intersection of these two boundaries represents the transition between the two deformation mechanisms, i.e. displacive deformation and diffusive deformation. The $\sigma \propto D^{3}$ boundary will shift to the right with lower strain rate, or vice versa. The crossover size scale, $D_{\mathrm{C}}\left(T_{\mathrm{H}}, \dot{\varepsilon}\right)$, will decrease with increasing $\dot{\varepsilon}$ and decreasing $T_{\mathrm{H}}$. In polycrystalline (nanocrystalline) metals, a transition from Hall-Petch strengthening to an inverse Hall-Petch relationship was predicted at very small grain sizes. But it has been difficult to experimentally confirm this expectation, because very small nanograins are unstable against grain growth such that samples in this grain size regime are usually unavailable for reliable mechanical tests. Here, using a Sn single crystal, we are able to demonstrate that indeed "smaller is much weaker", when the sample/grain size scale eventually drops into the regime where displacive plasticity is taken over by diffusive plasticity.

In summary, by quantitative testing of Sn samples inside a TEM with a unique load transducer, we have demonstrated that with decreasing sample size the normal "smaller is stronger" trend eventually switches to "smaller is much weaker". This transition arises from the competition between the displacive mechanisms that lead to Hall-Petch-type strengthening and the diffusive processes that mediate Coble diffusion softening (inverse Hall-Petch dependence on size). The dominance of diffusive plasticity as the underlying deformation mechanism at very small sample size is supported not only by the in situ observations but also by the match between the diffusivity calculated from an analytical model and that reported in literature for boundary diffusion. These findings may pave way for nanofabrication, for example precise sculpting of nanoscale metallic electrodes by surface diffusional creep at elevated temperatures, and have implications for life estimation of the shape stability of nanometallic components at room temperature.

\section{Methods}

The purity of the Sn bar we used to fabricate submicron sized samples is $99.95 \%$. For the compression tests, pillar-like samples with their diameters ranging from $170 \mathrm{~nm}$ to $500 \mathrm{~nm}$ and an aspect ratio of $\sim 2.5$ were machined using focused ion beam (FIB) The samples were tested inside a JEOL $2100 \mathrm{~F}$ TEM at $200 \mathrm{kV}$ with a Hysitron PI95 TEM PicoIndenter under constant displacement rate control. The engineering stress $\sigma$ was calculated as $\sigma=4 F / \pi d^{2}$, where $F$ is the measured force and $d$ is the contact diameter. The engineering strain $\varepsilon$ was calculated as $\varepsilon=\Delta L / L_{0}$, where $\Delta L$ is the compression displacement and $L_{0}$ is the initial pillar height. For more details see Ref. 23.

Metals illuminated by high-energy electron beam may suffer from radiation damage, including beam heating, ionization damage (radiolysis) and displacement damage $^{39}$. Under normal TEM imaging conditions, the beam-heating effects are negligible because of the good thermal conductivity of the $\mathrm{Sn}$ sample $\left(66.6 \mathrm{Wm}^{-1} \mathrm{~K}^{-1}\right.$ at $300 \mathrm{~K}^{40}$ ) and good thermal conduction in our experimental setup ${ }^{41-43}$. Given the electronic structure of metals, ionization damage is also small. Displacement damage is determined mainly by the threshold displacement energy (TDE) and atomic weight of the metal. The TDE of $\mathrm{Sn}$ is relatively low, at $\sim 12 \mathrm{eV}^{44}$. However, since the atomic number of $\mathrm{Sn}$ is large, the kinetic energy transferred from the $200 \mathrm{kV}$ (the operation voltage of the TEM) electrons is $\sim 4.4 \mathrm{eV}^{45,46}$, insufficient for displacement damage inside the bulk lattice. Therefore, it is the sample size induced transition from displacive-mechanism dominated plasticity to diffusional-mechanism dominated plasticity, rather than the electron beam effects, that plays the decisive role in the aforementioned change in deformation responses.

1. Coble, R. L. A Model for Boundary Diffusion Controlled Creep in Polycrystalline Materials. J. Appl. Phys. 34, 1679-1682 (1963).

2. Zhu, T. \& Li, J. Ultra-strength materials. Progress in Materials Science 55, 710-757 (2010).

3. Shan, Z. W. et al. Grain boundary-mediated plasticity in nanocrystalline nickel. Science 305, 654-657 (2004).

4. Wang, N., Wang, Z. R., Aust, K. T. \& Erb, U. Room temperature creep behavior of nanocrystalline nickel produced by an electrodeposition technique. Materials Science and Engineering A 237, 150-158 (1997).

5. Cai, B., Kong, Q. P., Lu, L. \& Lu, K. Interface controlled diffusional creep of nanocrystalline pure copper. Scripta Materialia 41, 755-759 (1999).

6. Youngdahl, C. J., Weertman, J. R., Hugo, R. C. \& Kung, H. H. Deformation behavior in nanocrystalline copper. Scripta Materialia 44, 1475-1478 (2001).

7. Shan, Z. W., Mishra, R. K., Asif, S. A., Warren, O. L. \& Minor, A. M. Mechanical annealing and source-limited deformation in submicrometre-diameter $\mathrm{Ni}$ crystals. NATURE MATERIALS 7, 115-119 (2008).

8. Kumar, K. S., Van Swygenhoven, H. \& Suresh, S. Mechanical behavior of nanocrystalline metals and alloys. Acta Materialia 51, 5743-5774 (2003).

9. Dao, M., Lu, L., Asaro, R., Dehosson, J. \& Ma, E. Toward a quantitative understanding of mechanical behavior of nanocrystalline metals. Acta Materialia 55, 4041-4065 (2007).

10. Strachan, D. R. et al. Clean electromigrated nanogaps imaged by transmission electron microscopy. Nano Letters 6, 441-444 (2006).

11. Joachim, C., Gimzewski, J. K. \& Aviram, A. Electronics using hybrid-molecular and mono-molecular devices. Nature 408, 541-548 (2000).

12. Strachan, D. R. et al. Real-time TEM imaging of the formation of crystalline nanoscale gaps. Physical Review Letters 100, 056805 (2008).

13. Reed, M. A., Zhou, C., Muller, C. J., Burgin, T. P. \& Tour, J. M. Conductance of a molecular junction. Science 278, 252-254 (1997)

14. Howatson, A. M., Lund, P. G. \& Todd, J. D. Engineering tables and data. 41 (Chapman and Hall, 1991).

15. Uchic, M. D., Dimiduk, D. M., Florando, J. N. \& Nix, W. D. Sample dimensions influence strength and crystal plasticity. Science 305, 986-989 (2004).

16. Wang, Z.-J. et al. Sample size effects on the large strain bursts in submicron aluminum pillars. Applied Physics Letters 100, (2012).

17. Wang, Z.-J., Shan, Z.-W., Li, J., Sun, J. \& Ma, E. Pristine-to-pristine regime of plastic deformation in submicron-sized single crystal gold particles. Acta Materialia 60, 1368-1377 (2012).

18. Bei, H. et al. Compressive strengths of molybdenum alloy micro-pillars prepared using a new technique. SCRIPTA MATERIALIA 57, 397-400 (2007).

19. Lu, Y., Song, J., Huang, J. Y. \& Lou, J. Surface dislocation nucleation mediated deformation and ultrahigh strength in sub-10-nm gold nanowires. Nano Res. 4, 1261-1267 (2011).

20. Zheng, H. et al. Discrete plasticity in sub-10-nm-sized gold crystals. Nature Communications 1, 144 (2010).

21. Kondo, Y. \& Takayanagi, K. Gold nanobridge stabilized by surface structure. Physical Review Letters 79, 3455-3458 (1997).

22. Greer, J. \& Nix, W. Nanoscale gold pillars strengthened through dislocation starvation. PHYSICAL REVIEW B 73, (2006).

23. Huang, L. et al. A new regime for mechanical annealing and strong sample-size strengthening in body centred cubic molybdenum. Nature Communications 2 , 547 (2011).

24. Qian, Y., Suzhi, L., Minor, A. M., Jun, S. \& Ma, E. High-strength titanium alloy nanopillars with stacking faults and enhanced plastic flow. Applied Physics Letters 100, 063109 (063104 pp.)-063109 (063104 pp.) (2012).

25. Kiener, D. \& Minor, A. M. Source-controlled yield and hardening of $\mathrm{Cu}(100)$ studied by in situ transmission electron microscopy. Acta Materialia 59, 1328-1337 (2011). 
26. Jia, Y., Mishra, R. K., Sachdev, A. K. \& Minor, A. M. In situ TEM compression testing of Mg and Mg-0.2wt.\% Ce single crystals. Scripta Materialia 64, 292-295 (2011).

27. Ford, J. M., Wheeler, J. \& Movchan, A. B. Computer simulation of grain-boundary diffusion creep. Acta Materialia 50, 3941-3955 (2002).

28. Zhang, W. \& Schneibel, J. H. The sintering of two particles by surface and grain boundary diffusion-a two-dimensional numerical study. Acta Metallurgica Et Materialia 43, 4377-4386 (1995).

29. Beere, W. \& Rutter, E. H. Stresses and Deformation at Grain Boundaries Philosophical Transactions of the Royal Society A: Mathematical, Physical and Engineering Sciences 288, 177-196 (1978).

30. Sun, P. H. \& Ohring, M. Tracer self-diffusion and electromigration in thin tin films. J. Appl. Phys. 47, 478-485 (1976).

31. Singh, P. \& Ohring, M. Tracer study of diffusion and electromigration in thin tin films. J. Appl. Phys. 56, 899-907 (1984).

32. Mishin, Y., Asta, M. \& Li, J. Atomistic modeling of interfaces and their impact on microstructure and properties. Acta Materialia 58, 1117-1151 (2010).

33. Einstein, A. The motion of elements suspended in static liquids as claimed in the molecular kinetic theory of heat. Annalen Der Physik 17, 549-560 (1905).

34. Sellers, M. S., Schultz, A. J., Basaran, C. \& Kofke, D. A. beta-Sn grain-boundary structure and self-diffusivity via molecular dynamics simulations. Physical Review B 81, 134111 (2010).

35. Cheng, J., Chen, S., Vianco, P. T. \& Li, J. C. M. Quantitative analysis for hillocks grown from electroplated Sn film. J. Appl. Phys. 107, (2010).

36. Ashby, M. F. A first report on deformation-mechanism maps. Acta Metallurgica 20, 887-897 (1972).

37. Suresh, S. \& Li, J. Material science: Deformation of the ultra-strong. Nature 456 716-717 (2008).

38. Yu, Q. et al. Strong crystal size effect on deformation twinning. Nature 463 335-338 (2010).

39. Egerton, R. F., Li, P. \& Malac, M. Radiation damage in the TEM and SEM. Micron 35, 399-409 (2004).

40. Haynes, W. M., Lide, D. R. \& Thomas, J. Bruno, P. D. CRC Handbook of Chemistry and Physics. (Taylor \& Francis, 2012).

41. Guo, H. et al. Tensile ductility and necking of metallic glass. Nature Materials 6 , 735-739 (2007).

42. Luo, J. H., Wu, F. F., Huang, J. Y., Wang, J. Q. \& Mao, S. X. Superelongation and Atomic Chain Formation in Nanosized Metallic Glass. Physical Review Letters 104, 215503 (2010).
43. Williams, D. B. \& Carter, C. B. Transmission Electron Microscopy: A Textbook for Materials Science. (Springer London, Limited, 2009).

44. Myhra, S. \& Gardiner, R. B. Evidence of an atomic displacement process in electron irradiated $\alpha$-tin. Physics Letters A 39, 405-406 (1972).

45. Hren, J., Goldstein, J. I. \& Joy, D. C. Introduction to Analytical Electron Microscopy. (Plenum Press, 1979).

46. Bradley, C. R. \& Zaluzec, N. J. ATOMIC SPUTTERING IN THE ANALYTICAL ELECTRON-MICROSCOPE. Ultramicroscopy 28, 335-338 (1989).

\section{Acknowledgments}

This work was supported by NSFC $(50925104,11132006$, and 51231005) and 973 Programs of China (2010CB631003, 2012CB619402). We also appreciate the support from the 111 Project of China (B06025). Both E.M. and J.L. carried out this work under an adjunct professorship at XJTU. J.L. also acknowledges support by NSF CMMI-0728069,

DMR-1008104 and DMR-1120901, and AFOSR FA9550-08-1-0325. We appreciate helpful discussions with Prof. Litao Sun, where similar phenomena were seen for Ag ligaments of even smaller size scale at room temperature.

\section{Author contributions}

Z.W.S. and J.L. designed the project. L.T. carried out the experiments and the data analysis. J.L., Z.W.S., L.T. and E.M. wrote the paper. J.S. provided the sample. All authors contributed to discussions of the results.

\section{Additional information}

Supplementary information accompanies this paper at http://www.nature.com/ scientificreports

Competing financial interests: The authors declare no competing financial interests. How to cite this article: Tian, L., Li, J., Sun, J., Ma, E. \& Shan, Z.-W. Visualizing size-dependent deformation mechanism transition in Sn. Sci. Rep. 3, 2113; DOI:10.1038/ srep02113 (2013)

(c) (i) (-) $€$ This work is licensed under a Creative Commons Attribution-

NonCommercial-NoDerivs 3.0 Unported license. To view a copy of this license, visit http://creativecommons.org/licenses/by-nc-nd/3.0 
SUBJECT AREAS: STRUCTURAL PROPERTIES MECHANICAL PROPERTIES CHARACTERIZATION AND ANALYTICAL TECHNIQUES METALS AND ALLOYS

SCIENTIFIC REPORTS: $3: 2113$ DOI: $10.1038 /$ srep02113 (2013)

Published:

3 July 2013

Updated:

5 August 2013

\section{ERRATUM: Visualizing size-dependent deformation mechanism transition in $\mathrm{Sn}$} Lin Tian, Ju Li, Jun Sun, Evan Ma \& Zhi-Wei Shan

Due to a technical error, the Supplementary Movies that accompany this study were not uploaded with the original Article. 